\title{
Imprimitive $Q$-polynomial Association Schemes*
}

HIROSHI SUZUKI

Department of Mathematics, International Christian University,

10-2, Osawa 3-chome, Mitaka-shi Tokyo 181, Japan

Received April 25, 1996; Revised January 13, 1997 hsuzuki@icu.ac.jp

Abstract. It is well known that imprimitive $P$-polynomial association schemes $\mathcal{X}=\left(X,\left\{R_{i}\right\}_{0 \leq i<d}\right)$ with $k_{1}>2$ are either bipartite or antipodal, i.e., intersection numbers satisfy either $a_{i}=0$ for all $i$, or $b_{i}=c_{d-i}$ for all $i \neq[d / 2]$. In this paper, we show that imprimitive $Q$-polynomial association schemes $\mathcal{X}=\left(X,\left\{R_{i}\right\}_{0<i<d}\right)$ with $d>6$ and $k_{1}^{*}>2$ are either dual bipartite or dual antipodal, i.e., dual intersection numbers satisfy either $a_{i}^{*}=0$ for all $i$, or $b_{i}^{*}=c_{d-i}^{*}$ for all $i \neq[d / 2]$.

Keywords: $Q$-polynomial, association scheme, imprimitivity, Krein parameters, distance-regular graph

\section{Introduction}

A $d$-class symmetric association scheme is a pair $\mathcal{X}=\left(X,\left\{R_{i}\right\}_{0 \leq i \leq d}\right)$, where $X$ is a finite set, each $R_{i}$ is a nonempty subset of $X \times X$ for $i=0,1, \ldots, d$ satisfying the following.

(i) $R_{0}=\{(x, x) \mid x \in X\}$

(ii) $\left\{R_{i}\right\}_{0 \leq i \leq d}$ is a partition of $X \times X$, i.e.,

$$
X \times X=R_{0} \cup R_{1} \cup \cdots \cup R_{d}, R_{i} \cap R_{j}=\emptyset \text { if } i \neq j .
$$

(iii) ${ }^{t} R_{i}=R_{i}$ for $i=0,1, \ldots, d$, where ${ }^{t} R_{i}=\left\{(y, x) \mid(x, y) \in R_{i}\right\}$.

(iv) There exist integers $p_{i, j}^{h}$ such that for all $x, y \in X$ with $(x, y) \in R_{h}$,

$$
p_{i, j}^{h}=\left|\left\{z \in X \mid(x, z) \in R_{i},(z, y) \in R_{j}\right\}\right| .
$$

We refer to $X$ as the vertex set of $\mathcal{X}$, and to the integers $p_{i, j}^{h}$ as the intersection numbers of $\mathcal{X}$.

Let $\mathcal{X}=\left(X,\left\{R_{i}\right\}_{0 \leq i \leq d}\right)$ be a symmetric association scheme. Let $\operatorname{Mat}_{X}(\boldsymbol{R})$ denote the algebra of matrices over the reals $\boldsymbol{R}$ with rows and columns indexed by $X$. The $i$-th adjacency matrix $A_{i} \in \operatorname{Mat}_{X}(\boldsymbol{R})$ of $\mathcal{X}$ is defined by

$$
\left(A_{i}\right)_{x y}=\left\{\begin{array}{ll}
1 & \text { if }(x, y) \in R_{i} \\
0 & \text { otherwise }
\end{array} \quad(x, y \in X) .\right.
$$

From $(i)-(i v)$ above, it is easy to see the following.

* This research was partially supported by the Grant-in-Aid for Scientific Research (No.06640075, No. 09640062), the Ministry of Education, Science and Culture, Japan. 
$(i)^{\prime} A_{0}=I$.

(ii $)^{\prime} \quad A_{0}+A_{1}+\cdots+A_{d}=J$, where $J$ is the all-1s matrix, and $A_{i} \circ A_{j}=\delta_{i, j} A_{i}$ for $0 \leq i, j \leq d$, where $\circ$ denotes the entry-wise matrix product.

$(\text { iii) })^{\prime}{ }^{t} A_{i}=A_{i}$ for $0 \leq i \leq d$.

$(i v)^{\prime} \quad A_{i} A_{j}=\sum_{h=0}^{d} p_{i, j}^{h} A_{h}$ for $0 \leq i, j \leq d$.

By the Bose-Mesner algebra of $\mathcal{X}$ we mean the subalgebra $\mathcal{M}$ of $\operatorname{Mat}_{X}(\boldsymbol{R})$ generated by the adjacency matrices $A_{0}, A_{1}, \ldots, A_{d}$. Observe by $(i v)^{\prime}$ above that the adjacency matrices form a basis for $\mathcal{M}$. Moreover, $\mathcal{M}$ consists of symmetric matrices and it is closed under $\circ$. In particular, $\mathcal{M}$ is commutative in both multiplications.

Since the algebra $\mathcal{M}$ consists of commutative symmetric matrices, there is a second basis $E_{0}, E_{1}, \ldots, E_{d}$ satisfying the following.

$(i)^{\prime \prime} \quad E_{0}=\frac{1}{|X|} J$.

$(\text { ii })^{\prime \prime} E_{0}+E_{1}+\cdots+E_{d}=I$, and $E_{i} E_{j}=\delta_{i, j} E_{i}$ for $0 \leq i, j \leq d$.

$(\text { iii) })^{\prime \prime} E_{i}=E_{i}$ for $0 \leq i \leq d$.

$(i v)^{\prime \prime} \quad E_{i} \circ E_{j}=\frac{1}{|X|} \sum_{h=0}^{d} q_{i, j}^{h} E_{h},(0 \leq i, j \leq d)$ for some real numbers $q_{i, j}^{h}$.

$E_{0}, E_{1}, \ldots, E_{d}$ are the primitive idempotents of the Bose-Mesner algebra. The parameters $q_{i, j}^{h}$ are called Krein parameters.

Conventionally, we assume $p_{i, j}^{h}$ and $q_{i, j}^{h}$ are zero if one of the indices $h, i, j$ is out of range $\{0,1, \ldots, d\}$ otherwise mentioned clearly.

A symmetric association scheme $\mathcal{X}=\left(X,\left\{R_{i}\right\}_{0 \leq i \leq d}\right)$ with respect to the ordering $R_{0}, R_{1}, \ldots, R_{d}$ of the relations is called a $P$-polynomial association scheme if the following conditions are satisfied.

(P1) $p_{i, j}^{h}=0$ if one of $h, i, j$ is greater than the sum of the other two.

(P2) $p_{i, j}^{h} \neq 0$ if one of $h, i, j$ is equal to the sum of the other two for $0 \leq h, i, j \leq d$.

In this case we write $c_{i}=p_{i-1,1}^{i}, a_{i}=p_{i, 1}^{i}, b_{i}=p_{i+1,1}^{i}$ and $k_{i}=p_{i, i}^{0}$ for $i=0,1, \ldots, d$.

A symmetric association scheme $\mathcal{X}=\left(X,\left\{R_{i}\right\}_{0 \leq i \leq d}\right)$ with respect to the ordering $E_{0}, E_{1}, \ldots, E_{d}$ of the primitive idempotents of the Bose-Mesner algebra is called a $Q$ polynomial association scheme if the following conditions are satisfied.

(Q1) $q_{i, j}^{h}=0$ if one of $h, i, j$ is greater than the sum of the other two.

(Q2) $q_{i, j}^{h} \neq 0$ if one of $h, i, j$ is equal to the sum of the other two for $0 \leq h, i, j \leq d$. 
In this case we write $c_{i}^{*}=q_{i-1,1}^{i}, a_{i}^{*}=q_{i, 1}^{i}, b_{i}^{*}=q_{i+1,1}^{i}$ and $k_{i}^{*}=q_{i, i}^{0}$ for $i=0,1, \ldots, d$. If $\mathcal{X}=\left(X,\left\{R_{i}\right\}_{0 \leq i \leq d}\right)$ is a $P$-polynomial association scheme with respect to the ordering $R_{0}, R_{1}, \ldots, R_{d}$, then the graph $\Gamma=\left(X, R_{1}\right)$ with vertex set $X$, edge set defined by $R_{1}$ becomes a distance-regular graph. In this case,

$$
R_{i}=\{(x, y) \in X \times X \mid \partial(x, y)=i\},
$$

where $\partial(x, y)$ denotes the distance between $x$ and $y$. Conversely, every distance-regular graph is obtained in this way.

$Q$-polynomial association schemes appear in design theory in connection with tight conditions, but it is not much studied compared with $P$-polynomial association schemes, though there are extensive studies of $P$ - and $Q$-polynomial association schemes.

A symmetric association scheme $\mathcal{X}=\left(X,\left\{R_{i}\right\}_{0 \leq i \leq d}\right)$ is said to be imprimitive if it satisfies one of the following equivalent conditions.

(A) By a suitable rearrangement of indices $1,2, \ldots, d$, there exists an index $s(0<s<d)$ such that $A_{i} A_{j}$ is a linear combination of $A_{0}, A_{1}, \ldots, A_{s}$ for all $i, j(0 \leq i, j \leq s)$.

$(E)$ By a suitable rearrangement of indices $1,2, \ldots, d$, there exists an index $t(0<t<d)$ such that $E_{i} \circ E_{j}$ is a linear combination of $E_{0}, E_{1}, \ldots, E_{t}$ for all $i, j(0 \leq i, j \leq t)$.

The imprimitivity of association schemes including the equivalence of the above definitions were first studied in [3]. We also refer the readers to sections 2.4, 2.9 and 3.6 in [1] and sections 2.4, 4.1 and 4.2 in [2].

The following is well known. See the references above.

Theorem 1 Let $\mathcal{X}=\left(X,\left\{R_{i}\right\}_{0 \leq i \leq d}\right)$ be an imprimitive P-polynomial association scheme with respect to the ordering $R_{0}, R_{1}, \ldots, R_{d}$ of the relations. If $k_{1}>2$, then one of the following holds.

(i) $a_{i}=0$ for all $i=0,1, \ldots, d$.

(ii) $b_{i}=c_{d-i}$ for all $i=0,1, \ldots, d$ except possibly for $i=[d / 2]$.

If the condition $(i)$ is satisfied, the scheme is called bipartite, and if the condition $(i i)$ is satisfied, it is called antipodal, by adopting the terminologies of the distance-regular graph associated with the $P$-polynomial structure.

The following is our main result in this paper.

Theorem 2 Let $\mathcal{X}=\left(X,\left\{R_{i}\right\}_{0 \leq i \leq d}\right)$ be an imprimitive Q-polynomial association scheme with respect to the ordering $E_{0}, E_{1}, \ldots, E_{d}$ of the primitive idempotents. If $d>6$ and $k_{1}^{*}>2$, then one of the following holds.

(i) $a_{i}^{*}=0$ for all $i=0,1, \ldots, d$.

(ii) $b_{i}^{*}=c_{d-i}^{*}$ for all $i=0,1, \ldots, d$ except possibly for $i=[d / 2]$. 
If the condition $(i)$ is satisfied, the scheme is called dual bipartite, and if the condition (ii) is satisfied, it is called dual antipodal. It is known that if $k_{1}^{*}=2$, then $\mathcal{X}$ is an ordinary polygon.

The proof of Theorem 1 is relatively easy and uses the inequalities based on the combinatorial structure of distance-regular graphs. We substitute that part by matrix identities to prove Theorem 2. These identities were used in Dickie's paper [5], which is a part of [4, Chapter 4].

\section{2. $P$-polynomial $C$-algebra}

We begin with a definition of $P$-polynomial $C$-algebra.

Let $d$ be a positive integer and let $c_{i+1}, a_{i}, b_{i-1}(i=0,1, \ldots, d)$ be real numbers satisfying the following.

(i) $a_{0}=b_{-1}=c_{d+1}=0$ and $c_{1}=1$.

(ii) $c_{i}+a_{i}+b_{i}=b_{0}=c_{d}+a_{d}$ for $i=1, \ldots, d-1$.

(iii) $b_{i} c_{i+1}>0$ for $i=0,1, \ldots, d-1$.

A $P$-polynomial $C$-algebra is an algebra over the reals $\boldsymbol{R}$ with basis $x_{0}, x_{1}, \ldots, x_{d}$, which satisfies the following.

$$
x_{0} x_{0}=x_{0}, \quad x_{1} x_{i}=b_{i-1} x_{i-1}+a_{i} x_{i}+c_{i+1} x_{i+1},(0 \leq i \leq d),
$$

where $x_{-1}$ and $x_{d+1}$ are indeterminates. Then $x_{i}$ can be written as a polynomial of $x_{1}$ of degree $i$ and $x_{0}=1$, the unit element in this algebra. Define constants $p_{i, j}^{h}$ by the following.

$$
x_{i} x_{j}=\sum_{h=0}^{d} p_{i, j}^{h} x_{h}, 0 \leq i, j \leq d .
$$

Since the algebra becomes commutative, $p_{i, j}^{h}=p_{j, i}^{h}$. Let $k_{i}=p_{i, i}^{0}, n=k_{0}+k_{1}+\cdots+k_{d}$, and $n e_{0}=x_{0}+x_{1}+\cdots+x_{d}$. Then it is easy to check by $(i)$ and $(i i)$ that $k_{1}=b_{0}$ and that $x_{1}\left(n e_{0}\right)=k_{1}\left(n e_{1}\right)$.

The algebra $\mathcal{M}=<x_{0}, x_{1}, \ldots, x_{d}>$ defined above becomes a $C$-algebra in the sense defined in [1, Section 2.5]. See also [1, Section 3.6] and (2) in the following lemma. In particular, $\mathcal{M}$ has another basis $\left\{e_{0}, e_{1}, \ldots, e_{d}\right\}$ consisting of primitive idempotents and the dual algebra $\mathcal{M}^{*}$ defined by $x_{i} \circ x_{j}=\delta_{i, j} x_{i}$ becomes a $C$-algebra with respect to the basis $n e_{0}=x_{0}+x_{1}+\cdots+x_{d}, n e_{1}, \ldots, n e_{d}$. Let

$$
e_{i} \circ e_{j}=\frac{1}{n} \sum_{h=0}^{d} q_{i, j}^{h} e_{h} .
$$

As the intersection numbers and the Krein parameters, by convention we assume the parameters $p_{i, j}^{h}$ and $q_{i, j}^{h}$ of $C$-algebras are zero if one of the indices $h, i, j$ is out of range $\{0,1, \ldots, d\}$. 
Lemma 1 Let $\mathcal{M}=<x_{0}, x_{1}, \ldots, x_{d}>$ be a P-polynomial C-algebra. Let $k_{i}=p_{i, i}^{0}$. Then the following hold.

(1) $p_{i+1, j}^{h} c_{i+1}=p_{i, j-1}^{h} b_{j-1}+p_{i, j}^{h}\left(a_{j}-a_{i}\right)+p_{i, j+1}^{h} c_{j+1}-p_{i-1, j}^{h} b_{i-1}$.

(2) $p_{i, j}^{0}=\delta_{i, j} k_{i}, k_{h} p_{i, j}^{h}=k_{i} p_{j, h}^{i}$ and $k_{i}>0$ for $i=0,1, \ldots$, d. In particular, $p_{i, j}^{h}=0$ if and only if $p_{h, j}^{i}=0$.

(3) $p_{i, j}^{h}=0$ if one of $h, i, j$ is greater than the sum of the other two.

(4) $p_{i, j}^{h} \neq 0$ if one of $h, i, j$ is equal to the sum of the other two for $0 \leq h, i, j \leq d$.

(5) $p_{i, h+1}^{i+h} c_{h+1}=p_{i, h}^{i+h}\left(a_{i}+\cdots+a_{i+h}-a_{1}-\cdots-a_{h}\right)$.

Proof: (1) Compute the coefficient of $x_{h}$ in the expression of $\left(x_{1} x_{i}\right) x_{j}=\left(x_{1} x_{j}\right) x_{i}$ by applying (1) and then (2), and we obtain the formula.

(2) First we prove that $c_{i+1} p_{i+1, j+1}^{0}=\delta_{i, j} b_{j} p_{i, j}^{0}$ for $0 \leq i \leq j \leq d-1$ by induction on $i$. If $i=0$, then this is obvious. Compute the coefficient of $x_{0}$ in the expression of $\left(x_{1} x_{i}\right) x_{j}=x_{i}\left(x_{1} x_{j}\right)$ in two ways. By induction hypothesis $p_{l, m}^{0}=0$ for $l<i+1, m$, we have $c_{i+1} p_{i+1, j+1}^{0}=b_{j} p_{i, j}^{0}$. Since $p_{i, j}^{0}=\delta_{i, j} p_{i, i}^{0}$, we have the assertion. Hence we have $p_{i, j}^{0}=\delta_{i, j} k_{i}$ and $k_{i} b_{i}=k_{i+1} c_{i+1}$. By our assumption $b_{i} c_{i+1}>0$, we have $k_{i}>0$ as $k_{0}=1$.

Next compute the coefficient of $x_{0}$ in the expression of $\left(x_{i} x_{j}\right) x_{h}=\left(x_{j} x_{h}\right) x_{i}$ in two ways using the formula $p_{i, j}^{0}=\delta_{i, j} k_{i}$ just shown above, and we obtain the second formula $k_{h} p_{i, j}^{h}=k_{i} p_{j, h}^{i}$.

(3) By (2), we may assume that $h>i+j$. Since $x_{i}$ is expressed as a polynomial of $x_{1}$ of degree $i$, we have the assertion.

(4) By (2), we may assume that $h=i+j$. Then by (1), $p_{i, j}^{i+j} c_{i}=p_{i-1, j+1}^{i+j} c_{j+1}$. Hence we have the assertion by induction on $i$.

(5) This follows by induction on $h$ using (1).

By definition, it is easy to see that the Bose-Mesner algebra $\mathcal{M}$ of a $P$-polynomial association scheme becomes a $P$-polynomial $C$-algebra with respect to the basis $A_{0}, A_{1}, \ldots, A_{d}$. Moreover, if we take $\circ$ product, the dual Bose-Mesner algebra $\mathcal{M}^{*}$ of $Q$-polynomial association scheme becomes a $P$-polynomial $C$-algebra with respect to the basis $|X| E_{0},|X| E_{1}$, $\ldots,|X| E_{d}$.

In both of these cases, the structure constants and Krein parameters are nonnegative, i.e., $p_{i, j}^{h} \geq 0$ and $q_{i, j}^{h} \geq 0$. The latter inequality is called the Krein condition.

Lemma 2 Let $\mathcal{M}=<x_{0}, x_{1}, \ldots, x_{d}>$ be a P-polynomial $C$-algebra. Suppose the structure constants $p_{i, j}^{h}$ are all nonnegative. Then the following hold.

(1) If $p_{i+1, j-1}^{h}=p_{i+1, j}^{h}=p_{i+1, j+1}^{h}=0$ for $0 \leq i<d$, then $p_{i, j}^{h}=p_{i+2, j}^{h}=0$.

(2) If $p_{l, j-l+i}^{h}=p_{l, j-l+i+1}^{h}=\cdots=p_{l, j+l-i}^{h}=0$ for $i \leq l$ and $0 \leq i<d$, then $p_{i, j}^{h}=p_{2 l-i, j}^{h}=0$. 
(3) For all $i, j$ with $0 \leq i, h, i+h \leq d, a_{i}=a_{i+1}=\cdots=a_{i+h}=0$ implies $a_{1}=\cdots=$ $a_{h}=0$.

(4) For all $h$ and $i$ with $0 \leq h, i, i+h \leq d$, the following hold.

(i) If $p_{i, i+h-1}^{h}=0$, then $a_{i} \leq a_{i+h}$. Moreover if $a_{i}=a_{i+h}$, then $p_{i+1, i+h}^{h}=0$.

(ii) If $p_{i+1, i+h}^{h}=0$, then $a_{i} \geq a_{i+h}$. Moreover if $a_{i}=a_{i+h}$, then $p_{i, i+h-1}^{h}=0$.

(iii) If $p_{i, i+h-1}^{h}=p_{i+1, i+h}^{h}=0$, then $a_{i}=a_{i+h}$.

(5) For all $h$ and $i$ with $0 \leq i \leq h \leq d$, the following hold.

(i) If $p_{i, h-i+1}^{h}=0$, then $a_{i} \leq a_{h-i}$. Moreover if $a_{i}=a_{h-i}$, then $p_{i+1, h-i}^{h}=0$.

(ii) If $p_{i+1, h-i}^{h}=0$, then $a_{i} \geq a_{h-i}$. Moreover if $a_{i}=a_{h-i}$, then $p_{i, h-i+1}^{h}=0$.

(iii) If $p_{i, h-i+1}^{h}=p_{i+1, h-i}^{h}=0$, then $a_{i}=a_{h-i}$.

Proof: (1) Replacing $i$ by $i+1$, by Lemma 1 (1) we have

$$
p_{i, j}^{h} b_{i}+p_{i+2, j}^{h} c_{i+2}=p_{i+1, j-1}^{h} b_{j-1}+p_{i+1, j}^{h}\left(a_{j}-a_{i+1}\right)+p_{i+1, j+1}^{h} c_{j+1} .
$$

Since $i<d$ by our assumption, $b_{i}>0$ and we have the assertion. Note that $b_{i}=p_{1, i+1}^{i}$ with $i<d$ is nonzero by the definition of $P$-polynomial $C$-algebra and it is nonnegative by our assumption.

(2) We prove the assertion by induction on $m=l-i$. If $l=i$, there is nothing to prove. Suppose the assertion holds for $m=l-i-1 \geq 0$. Then

$$
p_{i+1, j-1}^{h}=p_{i+1, j}^{h}=p_{i+1, j+1}^{h}=p_{2 l-i-1, j-1}^{h}=p_{2 l-i-1, j}^{h}=p_{2 l-i-1, j+1}^{h}=0 .
$$

By (1), we have $p_{i, j}^{h}=p_{2 l-i, j}^{h}=0$.

(3) This follows from Lemma 1 (4), (5) and the nonnegativity of the $a_{j}$ 's.

(4) Since $p_{i-1, i+h}^{h}=p_{i, i+h+1}^{h}=0$ by Lemma 1 (3), it follows from Lemma 1 (1) by setting $j=i+h$ that

$$
p_{i+1, i+h}^{h} c_{i+1}+p_{i, i+h}^{h} a_{i}=p_{i, i+h-1}^{h} b_{i+h-1}+p_{i, i+h}^{h} a_{i+h} .
$$

Since $p_{i, i+h}^{h} \neq 0$, we have the assertion.

(5) This is similar to (4). Consider the following.

$$
p_{i+1, h-i}^{h} c_{i+1}+p_{i, h-i}^{h} a_{i}=p_{i, h-i+1}^{h} c_{h-i+1}+p_{i, h-i}^{h} a_{h-i} .
$$

Lemma 3 Let $\mathcal{M}=<x_{0}, x_{1}, \ldots, x_{d}>$ be a P-polynomial C-algebra such that the structure constants $p_{i, j}^{h}$ are all nonnegative. Suppose for a positive integer $\alpha, p_{i, j \alpha}^{\alpha} \neq 0$ only if $i \equiv 0 \quad(\bmod \alpha)$. Then $p_{l, m}^{\alpha} \neq 0$ only if $l \equiv m$ or $-m \quad(\bmod \alpha)$.

Proof: It suffices to consider $p_{l, m}^{\alpha}$ with $0<m-l<\alpha$ by Lemma 1 (3). We may assume that $(2 i-1) \alpha<l+m<2 i \alpha$ or $2 i \alpha<l+m<(2 i+1) \alpha$. In the first case, there exists $0 \leq \beta \leq[\alpha / 2]-1$ such that $m=i \alpha-\beta$ or $i \alpha+\beta$ as $l<m$. Similarly, in the 
latter case, there exists $0 \leq \beta \leq[\alpha / 2]-1$ such that $l=i \alpha-\beta$ or $i \alpha+\beta$. Define $\gamma$ by the following: $l=(i-1) \alpha+\beta+\gamma$ in the first case and $m=(i+1) \alpha-\beta-\gamma$ in the latter. Since $0<m-l<\alpha$ and $m+l$ is in the corresponding range, in each case we have $1 \leq \gamma \leq \alpha-1$ and that $2 \beta+\gamma<\alpha$. Thus there are four cases.

(i) $l=(i-1) \alpha+\beta+\gamma$ and $m=i \alpha-\beta$.

(ii) $l=(i-1) \alpha+\beta+\gamma$ and $m=i \alpha+\beta$.

(iii) $l=i \alpha-\beta$ and $m=(i+1) \alpha-\beta-\gamma$.

(iv) $l=i \alpha+\beta$ and $m=(i+1) \alpha-\beta-\gamma$.

We apply Lemma $2(2)$. Since $p_{(i-1) \alpha+\gamma, i \alpha}^{\alpha}=\cdots=p_{(i-1) \alpha+2 \beta+\gamma, i \alpha}^{\alpha}=0, p_{l, m}^{\alpha}=0$ in the first two cases. Since $p_{i \alpha,(i+1) \alpha-2 \beta-\gamma}^{\alpha}=\cdots=p_{i \alpha,(i+1) \alpha-\gamma}^{\alpha}=0, p_{l, m}^{\alpha}=0$ in the last two cases.

The following is Proposition 6.2 in [1] but the description of it involves an error. Hence we restate the corrected version below. Note that we do not know if $b_{t}=c_{t+1}$ when $\alpha=2 t+1$.

Proposition 1 Let $\mathcal{M}=<x_{i} \mid 0 \leq i \leq d>$ be a P-polynomial $C$-algebra with respect to the basis $x_{0}, x_{1}, \ldots, x_{d}$. Assume $p_{i, j}^{h} \geq 0$ and $q_{i, j}^{h} \geq 0$ for all $h, i, j$. Let $\left\langle x_{\beta}\right| \beta \in T>$ be a proper $C$-subalgebra of $\mathcal{M}$. Then

$$
T=\{0, \alpha, 2 \alpha, 3 \alpha, \ldots\} \text { for some } \alpha \in\left\{2, d, \frac{d}{s}, \frac{2 d+1}{2 s+1}, \frac{2 d}{2 s+1}\right\} .
$$

Let the following be the array of defining parameters,

$$
\left\{\begin{array}{c}
c_{i} \\
a_{i} \\
b_{i}
\end{array}\right\}=\left\{\begin{array}{cccccc}
* & 1 & c_{2} & \cdots & c_{d-1} & c_{d} \\
0 & a_{1} & a_{2} & \cdots & a_{d-1} & a_{d} \\
b_{0} & b_{1} & b_{2} & \cdots & b_{d-1} & *
\end{array}\right\} .
$$

Then $\mathcal{M}$ has a C-subalgebra $<x_{\beta} \mid \beta \in T>$ with (i) $\alpha=2$, (ii) $\alpha=d$, (iii) $\alpha \in\left\{\frac{d}{s}, \frac{2 d+1}{2 s+1}, \frac{2 d}{2 s+1}\right\}$ respectively if and only if the following hold.

(i) $a_{2}=a_{4}=\cdots=0$ and $a_{1}=a_{3}=\cdots$.

(ii) $b_{i}=c_{d-i}$ for all $i$ except possibly for $i=[d / 2]$.

(iii) The parameters $c_{h}, a_{h}, b_{h}$ satisfy the following for $0 \leq h \leq d-1 . b_{i}=c_{\alpha-i}=$ $b_{j \alpha+i}=c_{(j+1) \alpha-i}$ for all $1 \leq i \leq \alpha-1$ and $1 \leq j$ except for $i=[\alpha / 2], a_{i}=a_{\alpha-i}=$ $a_{j \alpha+i}=a_{(j+1) \alpha-i}$ for all $0 \leq i \leq \alpha$ and $1 \leq j$ except for $i=[\alpha / 2],[(\alpha+1) / 2]$ with odd $\alpha$. Moreover,

$$
\left(c_{d}, a_{d}\right)= \begin{cases}\left(b_{0}, 0\right) & \text { if } \alpha=\frac{d}{s} \\ \left(c_{(\alpha-1) / 2}, a_{(\alpha-1) / 2}+b_{(\alpha-1) / 2}\right) & \text { if } \alpha=\frac{2 d+1}{2 s+1} \\ \left(c_{\alpha / 2}+b_{\alpha / 2}, a_{\alpha / 2}\right) & \text { if } \alpha=\frac{2 d}{2 s+1}\end{cases}
$$

Note that (i) and (ii) are special cases of (iii) for $\alpha=2$ and $\alpha=d$, respectively. 


\section{Vanishing Conditions of Krein Parameters}

Only a few restrictions of the Krein parameters $q_{i, j}^{h}$ of symmetric association schemes are known except those derived algebraically using Lemma 1 . We first list them in the following.

Proposition 2 Let $\mathcal{X}=\left(X,\left\{R_{i}\right\}_{0 \leq i \leq d}\right)$ be a symmetric association scheme. Let $E_{0}, E_{1}$, $\ldots, E_{d}$ be primitive idempotents and let $q_{i, j}^{h}$ be the Krein parameters. Then the following hold.

(1) $q_{i, j}^{h} \geq 0$ for all $0 \leq h, i, j \leq d$.

(2) For $0 \leq h, i, j \leq d$, we have

$$
q_{i, j}^{h}=0 \Leftrightarrow \sum_{u \in X}\left(E_{h}\right)_{u x}\left(E_{i}\right)_{u y}\left(E_{j}\right)_{u z}=0 \text { for all } x, y, z \in X .
$$

Proposition 2 (1) is known as Krein condition and (2) is in [3]. See also [1, Theorem 2.3.8, Proposition 2.8.3].

Lemma 4 Let $\mathcal{X}=\left(X,\left\{R_{i}\right\}_{0 \leq i \leq d}\right)$ be a symmetric association scheme. Let $E_{0}, E_{1}, \ldots, E_{d}$ be primitive idempotents and let $q_{i, j}^{h}$ be the Krein parameters. Suppose $\left\{i \mid q_{j, k}^{i} q_{l, m}^{i} \neq 0\right\} \subset\{h\}$. Then for all integers $0 \leq h, i, j, k, l, m \leq d$ and all vertices $a, a^{\prime}, b, b^{\prime}$, the following hold.

(1) $\sum_{e \in X}\left(E_{j}\right)_{e a}\left(E_{k}\right)_{e a^{\prime}}\left(E_{l}\right)_{e b}\left(E_{m}\right)_{e b}=\frac{q_{l, m}^{h}}{|X|} \sum_{e \in X}\left(E_{j}\right)_{e a}\left(E_{k}\right)_{e a^{\prime}}\left(E_{h}\right)_{e b}$.

(2) $\sum_{e \in X}\left(E_{j}\right)_{e a}\left(E_{k}\right)_{e a^{\prime}}\left(E_{l}\right)_{e b}\left(E_{m}\right)_{e b^{\prime}}=\sum_{e, e^{\prime} \in X}\left(E_{j}\right)_{e a}\left(E_{k}\right)_{e a^{\prime}}\left(E_{h}\right)_{e e^{\prime}}\left(E_{l}\right)_{e^{\prime} b}\left(E_{m}\right)_{e^{\prime} b^{\prime}}$

Proof: (1) By Proposition 2 (2), we have

$$
\begin{aligned}
& \sum_{e \in X}\left(E_{j}\right)_{e a}\left(E_{k}\right)_{e a^{\prime}}\left(E_{l}\right)_{e b}\left(E_{m}\right)_{e b} \\
& =\sum_{e \in X}\left(E_{j}\right)_{e a}\left(E_{k}\right)_{e a^{\prime}}\left(E_{l} \circ E_{m}\right)_{e b} \\
& =\frac{1}{|X|} \sum_{i=0}^{d} q_{l, m}^{i} \sum_{e \in X}\left(E_{j}\right)_{e a}\left(E_{k}\right)_{e a^{\prime}}\left(E_{i}\right)_{e b} \\
& =\frac{q_{l, m}^{h}}{|X|} \sum_{e \in X}\left(E_{j}\right)_{e a}\left(E_{k}\right)_{e a^{\prime}}\left(E_{h}\right)_{e b} .
\end{aligned}
$$


(2) Since $I=E_{0}+E_{1}+\cdots+E_{d}$, similarly we have

$$
\begin{aligned}
& \sum_{e \in X}\left(E_{j}\right)_{e a}\left(E_{k}\right)_{e a^{\prime}}\left(E_{l}\right)_{e b}\left(E_{m}\right)_{e b^{\prime}} \\
& =\sum_{e, e^{\prime} \in X}\left(E_{j}\right)_{e a}\left(E_{k}\right)_{e a^{\prime}}(I)_{e e^{\prime}}\left(E_{l}\right)_{e^{\prime} b}\left(E_{m}\right)_{e^{\prime} b^{\prime}} \\
& =\sum_{i=0}^{d} \sum_{e, e^{\prime} \in X}\left(E_{j}\right)_{e a}\left(E_{k}\right)_{e a^{\prime}}\left(E_{i}\right)_{e e^{\prime}}\left(E_{l}\right)_{e^{\prime} b}\left(E_{m}\right)_{e^{\prime} b^{\prime}} \\
& =\sum_{i=0}^{d} \sum_{e^{\prime} \in X}\left(\sum_{e \in X}\left(E_{j}\right)_{e a}\left(E_{k}\right)_{e a^{\prime}}\left(E_{i}\right)_{e e^{\prime}}\right)\left(E_{l}\right)_{e^{\prime} b}\left(E_{m}\right)_{e^{\prime} b^{\prime}} \\
& =\sum_{i=0}^{d} \sum_{e \in X}\left(E_{j}\right)_{e a}\left(E_{k}\right)_{e a^{\prime}}\left(\sum_{e^{\prime} \in X}\left(E_{i}\right)_{e e^{\prime}}\left(E_{l}\right)_{e^{\prime} b}\left(E_{m}\right)_{e^{\prime} b^{\prime}}\right) .
\end{aligned}
$$

Comparing the last two expressions using Proposition $2(2)$ we have the right hand side of (2) by our assumption.

Proposition 3 Let $\mathcal{X}=\left(X,\left\{R_{i}\right\}_{0 \leq i \leq d}\right)$ be a Q-polynomial association scheme with respect to the ordering $E_{0}, E_{1}, \ldots, E_{d}$ of primitive idempotents. Suppose that

$$
\left\{l \mid q_{j, h+i}^{l} q_{i-j, h+j}^{l} \neq 0\right\} \subset\{h+i-j\} .
$$

Then for $h \geq 0, i \geq j \geq 1$ with $h+i+j \leq d, q_{i, h+j}^{h+i}=0$ implies that $q_{j, h+j}^{h+j}=0$.

Proof: Since $q_{i, h+j}^{h+i}=0$, by Proposition 2,

$$
\begin{aligned}
0 & =\frac{q_{j, i-j}^{i}}{|X|} \sum_{u \in X}\left(E_{h+i}\right)_{u x}\left(E_{i}\right)_{u y}\left(E_{h+j}\right)_{u z} \\
& =\sum_{u \in X}\left(\frac{q_{j, i-j}^{i}}{|X|}\left(E_{i}\right)_{u y}\right)\left(E_{h+i}\right)_{u x}\left(E_{h+j}\right)_{u z} \\
& =\sum_{u \in X}\left(\left(E_{j} \circ E_{i-j}\right) E_{i}\right)_{u y}\left(E_{h+i}\right)_{u x}\left(E_{h+j}\right)_{u z} \\
& =\sum_{u \in X} \sum_{v \in X}\left(E_{j}\right)_{u v}\left(E_{i-j}\right)_{u v}\left(E_{i}\right)_{v y}\left(E_{h+i}\right)_{u x}\left(E_{h+j}\right)_{u z} \\
& =\sum_{v \in X}\left(E_{i}\right)_{v y}\left(\sum_{u \in X}\left(E_{j}\right)_{u v}\left(E_{h+i}\right)_{u x}\left(E_{i-j}\right)_{u v}\left(E_{h+j}\right)_{u z}\right) .
\end{aligned}
$$

Since $\left\{l \mid q_{j, h+i}^{l} q_{i-j, h+j}^{l} \neq 0\right\} \subset\{h+i-j\}$, by Lemma 4 (2),

$$
=\sum_{u \in X} \sum_{v \in X} \sum_{w \in X}\left(E_{i}\right)_{v y}\left(E_{j}\right)_{u v}\left(E_{h+i}\right)_{u x}\left(E_{h+i-j}\right)_{u w}\left(E_{i-j}\right)_{w v}\left(E_{h+j}\right)_{w z} .
$$


Since this holds for arbitrary $x, y, z$, we have

$$
\begin{aligned}
0= & \sum_{x, y, z \in X}\left(E_{h+i+j}\right)_{x y}\left(E_{h+j}\right)_{y z}\left(E_{j}\right)_{x z} \times \\
& \sum_{u, v, w \in X}\left(E_{i}\right)_{v y}\left(E_{j}\right)_{u v}\left(E_{h+i}\right)_{u x}\left(E_{h+i-j}\right)_{u w}\left(E_{i-j}\right)_{w v}\left(E_{h+j}\right)_{w z} \\
= & \sum_{y, z, v, w \in X}\left(E_{h+j}\right)_{y z}\left(E_{i}\right)_{v y}\left(E_{i-j}\right)_{w v}\left(E_{h+j}\right)_{w z} \times \\
& \sum_{x, u \in X}\left(E_{h+i+j}\right)_{x y}\left(E_{j}\right)_{x z}\left(E_{h+i}\right)_{u x}\left(E_{j}\right)_{u v}\left(E_{h+i-j}\right)_{u w} .
\end{aligned}
$$

Since $\left\{l \mid q_{h+i+j, j}^{l} q_{j, h+i-j}^{l} \neq 0\right\} \subset\{h+i\}$, by Lemma 4 (2) we have

$$
\begin{aligned}
= & \sum_{y, z, v, w \in X}\left(E_{h+j}\right)_{y z}\left(E_{i}\right)_{v y}\left(E_{i-j}\right)_{w v}\left(E_{h+j}\right)_{w z} \times \\
& \sum_{x \in X}\left(E_{h+i+j}\right)_{x y}\left(E_{j}\right)_{x z}\left(E_{j}\right)_{x v}\left(E_{h+i-j}\right)_{x w} \\
= & \sum_{x, z, w \in X}\left(E_{h+j}\right)_{w z}\left(E_{j}\right)_{x z}\left(E_{h+i-j}\right)_{x w} \times \\
& \sum_{y, v \in X}\left(E_{h+i+j}\right)_{y x}\left(E_{h+j}\right)_{y z}\left(E_{i}\right)_{y v}\left(E_{j}\right)_{x v}\left(E_{i-j}\right)_{w v} .
\end{aligned}
$$

Since $\left\{l \mid q_{h+i+j, h+j}^{l} q_{j, i-j}^{l} \neq 0\right\} \subset\{i\}$, by Lemma 4 (2) we have

$$
\begin{aligned}
= & \sum_{x, z, w \in X}\left(E_{h+j}\right)_{w z}\left(E_{j}\right)_{x z}\left(E_{h+i-j}\right)_{x w} \times \\
& \sum_{y \in X}\left(E_{h+i+j}\right)_{y x}\left(E_{h+j}\right)_{y z}\left(E_{j}\right)_{x y}\left(E_{i-j}\right)_{w y} \\
= & \sum_{x, z, w \in X}\left(E_{h+j}\right)_{w z}\left(E_{j}\right)_{x z}\left(E_{h+i-j}\right)_{x w} \times \\
& \sum_{y \in X}\left(E_{h+j}\right)_{y z}\left(E_{i-j}\right)_{y w}\left(E_{j}\right)_{y x}\left(E_{h+i+j}\right)_{y x} .
\end{aligned}
$$

Since $\left\{l \mid q_{h+j, i-j}^{l} q_{j, h+i+j}^{l} \neq 0\right\} \subset\{h+i\}$, by Lemma 4 (1) we have

$$
\begin{aligned}
= & \frac{q_{j, h+i+j}^{h+i}}{|X|} \sum_{x, z, w \in X}\left(E_{h+j}\right)_{w z}\left(E_{j}\right)_{x z}\left(E_{h+i-j}\right)_{x w} \times \\
& \sum_{y \in X}\left(E_{h+j}\right)_{y z}\left(E_{i-j}\right)_{y w}\left(E_{h+i}\right)_{y x} \\
= & \frac{q_{j, h+i+j}^{h+i}}{|X|} \sum_{y, z \in X}\left(E_{h+j}\right)_{y z} \times \\
& \sum_{x, w \in X}\left(E_{h+i}\right)_{x y}\left(E_{j}\right)_{x z}\left(E_{h+i-j}\right)_{x w}\left(E_{i-j}\right)_{w y}\left(E_{h+j}\right)_{w z} .
\end{aligned}
$$


Since $\left\{l \mid q_{h+i, j}^{l} q_{i-j, h+j}^{l} \neq 0\right\} \subset\{h+i-j\}$, by Lemma 4 (2), we have

$$
\begin{aligned}
& =\frac{q_{j, h+i+j}^{h+i}}{|X|} \sum_{y, z \in X}\left(E_{h+j}\right)_{y z} \sum_{x \in X}\left(E_{h+i}\right)_{x y}\left(E_{j}\right)_{x z}\left(E_{i-j}\right)_{x y}\left(E_{h+j}\right)_{x z} \\
& =\frac{q_{j, h+i+j}^{h+i}}{|X|} \sum_{x, z \in X}\left(E_{j}\right)_{x z}\left(E_{h+j}\right)_{x z} \sum_{y \in X}\left(E_{i-j}\right)_{x y}\left(E_{h+i}\right)_{x y}\left(E_{h+j}\right)_{y z} \\
& =\frac{q_{j, h+i+j}^{h+i}}{|X|} \sum_{x, z \in X}\left(E_{j}\right)_{x z}\left(E_{h+j}\right)_{x z} \sum_{y \in X}\left(\left(E_{i-j}\right) \circ\left(E_{h+i}\right)\right)_{x y}\left(E_{h+j}\right)_{y z} \\
& =\frac{q_{j, h+i+j}^{h+i} q_{i-j, h+i}^{h+j}}{|X|^{2}} \sum_{x, z \in X}\left(E_{j}\right)_{x z}\left(E_{h+j}\right)_{x z}\left(E_{h+j}\right)_{x z} \\
& =\frac{q_{j, h+i+j}^{h+i} q_{i-j, h+i}^{h+j}}{|X|^{2}} \sum_{x \in X}\left(\sum_{z \in X}\left(\left(E_{j}\right) \circ\left(E_{h+j}\right)\right)_{x z}\left(E_{h+j}\right)_{z x}\right) \\
& =\frac{q_{j, h+i+j}^{h+i} q_{i-j, h+i}^{h+j} q_{j, h+j}^{h+j}}{|X|^{3}} \sum_{x \in X}\left(E_{h+j}\right)_{x x} .
\end{aligned}
$$

Since $E_{h+j}$ is a nonzero idempotent,

$$
\sum_{x \in X}\left(E_{h+j}\right)_{x x}=\operatorname{trace}\left(E_{h+j}\right)=\operatorname{rank}\left(E_{h+j}\right) \neq 0 .
$$

Moreover, $q_{j, h+i+j}^{h+i} \neq 0$ and $q_{i-j, h+i}^{h+j} \neq 0$ by $(Q 2)$. Hence $q_{j, h+j}^{h+j}=0$.

Corollary 1 Let $\mathcal{X}=\left(X,\left\{R_{i}\right\}_{0 \leq i \leq d}\right)$ be a $Q$-polynomial association scheme with respect to the ordering $E_{0}, E_{1}, \ldots, E_{d}$ of primitive idempotents.

(1) For $h \geq 0, i \geq 1$ with $h+i+1 \leq d$,

$$
q_{i, h+1}^{h+i}=q_{1, h+i}^{h+i}=0 \text { implies that } q_{1, h+1}^{h+1}=0 .
$$

(2) For $h \geq 0, i \geq 2$ with $h+i+2 \leq d$,

$$
q_{i, h+2}^{h+i}=q_{2, h+i}^{h+i}=q_{2, h+i-1}^{h+i}=0 \text { implies that } q_{2, h+2}^{h+2}=0 .
$$

Proof: (1) Since $q_{1, h+i}^{h+i}=0$, by (Q2) we have the following.

$$
\left\{l \mid q_{1, h+i}^{l} q_{i-1, h+1}^{l} \neq 0\right\} \subset\{h+i-1\} .
$$

Hence we have the assertion from Proposition 3 by setting $j=1$.

(2) Since $q_{2, h+i}^{h+i}=q_{2, h+i-1}^{h+i}$, by (Q2) we have the following.

$$
\left\{l \mid q_{2, h+i}^{l} q_{i-2, h+2}^{l} \neq 0\right\} \subset\{h+i-2\} .
$$


Hence we have the assertion from Proposition 3 by setting $j=2$.

By setting $h=0$ in Corollary 1, we obtain the result of G. A. Dickie in [4, 5]. Hence the proposition is a generalization of it. The following result for $P$-polynomial association schemes is not used elsewhere in this paper but it is the dual of the result above, which can be proved very similarly. The proof suggests a possible way to find vanishing conditions and its proof in $Q$-polynomial association schemes.

Proposition 4 Let $\mathcal{X}=\left(X,\left\{R_{i}\right\}_{0 \leq i \leq d}\right)$ be a P-polynomial association scheme with respect to the ordering $R_{0}, R_{1}, \ldots, R_{d}$ of relations. Suppose that

$$
\left\{l \mid p_{j, h+i}^{l} p_{i-j, h+j}^{l} \neq 0\right\} \subset\{h+i-j\} .
$$

Then for $h \geq 0, i \geq j \geq 1$, and $h+i+j \leq d, p_{i, h+j}^{h+i}=0$ implies that $p_{j, h+j}^{h+j}=0$.

Proof: Suppose $p_{j, h+j}^{h+j} \neq 0$. Then there are vertices $\alpha, \beta, \gamma \in X$ such that

$$
(\alpha, \beta),(\alpha, \gamma) \in R_{h+j} \text { and }(\beta, \gamma) \in R_{j} .
$$

Since $p_{i-j, h+i}^{h+j} \neq 0$ by $(P 2)$, there exists a vertex $\delta \in X$ such that $(\alpha, \delta) \in R_{i-j}$ and $(\delta, \beta) \in R_{h+i}$. Consider two triples $(\delta, \beta, \gamma)$ and $(\delta, \alpha, \gamma)$. Since $\left\{l \mid p_{h+i, j}^{l} p_{i-j, h+j}^{l} \neq\right.$ $0\} \subset\{h+i-j\},(\delta, \gamma) \in R_{h+i-j}$. Since $(\beta, \delta) \in R_{h+i}$ and $p_{h+i+j, j}^{h+i} \neq 0$ by $(P 2)$, there exists a vertex $\epsilon \in X$ such that $(\beta, \epsilon) \in R_{h+i+j}$ and $(\epsilon, \delta) \in R_{j}$. Consider two triples $(\epsilon, \beta, \alpha)$ and $(\epsilon, \delta, \alpha)$. Since $\left\{l \mid p_{h+i+j, h+j}^{l} p_{j, i-j}^{l} \neq 0\right\} \subset\{i\}$, we have $(\epsilon, \alpha) \in R_{i}$.

Next consider two triples $(\epsilon, \beta, \gamma)$ and $(\epsilon, \delta, \gamma)$. Since $\left\{l \mid p_{h+i+j, j}^{l} p_{j, h+i-j}^{l} \neq 0\right\} \subset$ $\{h+i\}$, we have $(\epsilon, \gamma) \in R_{h+i}$. Finally consider a triple $(\epsilon, \alpha, \gamma)$. Since

$$
(\epsilon, \gamma) \in R_{h+i},(\epsilon, \alpha) \in R_{i}, \text { and }(\alpha, \gamma) \in R_{h+j},
$$

$p_{i, h+j}^{h+i} \neq 0$, which is a contradiction.

\section{Proof of Main Theorem}

In this section, we prove the following result. It is obvious that Theorem 2 is a direct consequence of it.

Theorem 3 Let $\mathcal{X}=\left(X,\left\{R_{i}\right\}_{0 \leq i \leq d}\right)$ be a Q-polynomial association scheme with respect to the ordering $E_{0}, E_{1}, \ldots, E_{d}$ of the primitive idempotents. Suppose $\mathcal{X}$ is imprimitive. Or more precisely, suppose the linear span of $\left\{E_{i} \mid i \in T\right\}$ is closed under o product for some proper subset $T$ of $\{0,1, \ldots, d\}$ with $T \neq\{0\}$. In addition, assume that $k_{1}^{*}>2$. Then $T=\{0, \alpha, 2 \alpha, 3 \alpha, \ldots\}$ for some $\alpha \geq 2$, and one of the following holds.

(i) $\alpha=2$ and $a_{i}^{*}=0$ for all $i$.

(ii) $\alpha=d$ and $b_{i}^{*}=c_{d-i}^{*}$ for all $i=0,1, \ldots$, d except possibly for $i=[d / 2]$. 
(iii) $d=4, \alpha=3$, and the parameters satisfy the following conditions.

$$
\left\{\begin{array}{l}
c_{i}^{*} \\
a_{i}^{*} \\
b_{i}^{*}
\end{array}\right\}=\left\{\begin{array}{ccccc}
* & 1 & c_{2}^{*} & c_{3}^{*} & 1 \\
0 & 0 & a_{2}^{*} & 0 & k^{*}-1 \\
k^{*} & k^{*}-1 & 1 & b_{3}^{*} & *
\end{array}\right\} .
$$

(iv) $d=6, \alpha=3$, and the parameters satisfy the following conditions.

$$
\left\{\begin{array}{l}
c_{i}^{*} \\
a_{i}^{*} \\
b_{i}^{*}
\end{array}\right\}=\left\{\begin{array}{ccccccc}
* & 1 & c_{2}^{*} & c_{3}^{*} & 1 & c_{5}^{*} & k^{*} \\
0 & 0 & a_{4}^{*}+a_{5}^{*} & 0 & a_{4}^{*} & a_{5}^{*} & 0 \\
k^{*} & k^{*}-1 & 1 & b_{3}^{*} & b_{4}^{*} & 1 & *
\end{array}\right\} .
$$

It is not difficult to see from Proposition 1 that if one of the conditions $(i)-(i v)$ of the theorem above holds, then the linear span of $\left\{E_{i} \mid i \in T\right\}$ is closed under $\circ$ product, where $T=\{0, \alpha, 2 \alpha, 3 \alpha, \ldots\}$. In particular, the association scheme $\mathcal{X}$ is imprimitive.

Throughout this section assume the following:

$\mathcal{X}=\left(X,\left\{R_{i}\right\}_{0 \leq i \leq d}\right)$ is a Q-polynomial association scheme with respect to the ordering $E_{0}, E_{1}, \ldots, E_{d}$ of the primitive idempotents such that the linear span of $\left\{E_{i} \mid i \in T\right\}$ is closed under $\circ$ product for some proper subset $T$ of $\{0,1, \ldots, d\}$ with $T \neq\{0\}$.

Under the assumption above, $\mathcal{M}^{*}=<|X| E_{0},|X| E_{1}, \ldots,|X| E_{d}>$ with $\circ$ product is a $P$-polynomial $C$-algebra with nonnegative $p_{i, j}^{h}$ and $q_{i, j}^{h}$. Hence we can apply Proposition 1. In particular, we have the following two lemmas as direct consequences.

Lemma 5 (1) $T=\{0, \alpha, 2 \alpha, 3 \alpha, \ldots\}$ for some $\alpha \geq 2$.

(2) Let $\beta=[\alpha / 2]$. Then $d \equiv 0$ or $\beta \quad(\bmod \alpha)$.

Lemma 6 Let $\beta=[\alpha / 2]$. Then the following hold.

(1) $q_{l, m}^{\alpha} \neq 0$ only if $l \equiv m$ or $-m \quad(\bmod \alpha)$.

(2) $q_{\alpha-h+1, h}^{\alpha}=0$, unless $\alpha=2 \beta+1$ with $h=\beta+1$.

(3) $q_{\alpha+h-1, h}^{\alpha}=0$, unless $\alpha=2 \beta+1$ with $h \equiv \beta+1 \quad(\bmod \alpha)$.

(4) Suppose $\alpha>2$ and $2 \leq h \leq \alpha$. Then $q_{\alpha-h+2, h}^{\alpha} \neq 0$, unless $\alpha=2 \beta$ with $h=\beta+1$.

Proof: (1) By Proposition 2, $q_{i, j}^{h} \geq 0$ for all $h, i$ and $j$. Hence this is a direct consequence of Lemma 3.

(2) By $(1)$ we have that $2 h-1 \equiv 0 \quad(\bmod \alpha)$, if the value is not zero. Hence $\alpha$ is odd and $h=\beta+1$.

(3) This is similar to (2).

(4) By $(1)$ we have that $2 h-2 \equiv 0 \quad(\bmod \alpha)$, if the value is not zero. Since $2 \leq h \leq \alpha$, $\alpha$ is even and $h=\beta+1$. 
Lemma 7 If $\alpha<d$, then $a_{i}^{*}=0$ for all $i=0,1, \ldots, \alpha$ except for $i=\beta+1$ with $\alpha=2 \beta+1$.

Proof: By Lemma $6(2), q_{\alpha-i+1, i}^{\alpha}=0$, unless $\alpha=2 \beta+1$ with $i=\beta+1$. Since $q_{1, \alpha}^{\alpha}=0$ by our assumption, we have $a_{i}^{*}=q_{1, i}^{i}=0$ by Corollary 1 (1) as desired.

Lemma 8 The following hold.

(1) Suppose $\alpha=2 \beta$. Then for each $0 \leq h \leq \alpha$ and $i \geq 0$ with $0 \leq h+i \alpha \leq d$, $a_{h}^{*}=a_{h+i \alpha}^{*}$.

(2) Suppose $\alpha=2 \beta+1$. Then for each $0 \leq h \leq \alpha$ and $i \geq 0$ with $0 \leq h+i \alpha \leq d$, $a_{h}^{*}=a_{h+i \alpha}^{*}$ unless $h=\beta, \beta+1$. Moreover, $a_{(i-1) \alpha+\beta}^{*} \leq a_{i \alpha+\beta}^{*}$, and $a_{(i-1) \alpha+\beta+1}^{*} \geq$ $a_{i \alpha+\beta+1}^{*}$.

Proof: By Lemma 6 (3), we have that $q_{\alpha+h-1, h}^{\alpha}=0$, unless $\alpha=2 \beta+1$ with $h \equiv \beta+1$ $(\bmod \alpha)$.

(1) Suppose $\alpha=2 \beta$. Then $q_{\alpha+h-1, h}^{\alpha}=0$ for every $h$. Hence by Lemma 2 (4)(iii), we have that $a_{h}^{*}=a_{h+i \alpha}^{*}$, for each $0 \leq h \leq \alpha$ and $i \geq 0$ with $0 \leq h+i \alpha \leq d$.

(2) Suppose $\alpha=2 \beta+1$. Then $q_{\alpha+h-1, h}^{\alpha}=0$, unless $h \equiv \beta+1 \quad(\bmod \alpha)$. Hence by Lemma 2 (4), $a_{h}^{*}=a_{h+i \alpha}^{*}$ unless $h=\beta, \beta+1$, for each $0 \leq h \leq \alpha$ and $i \geq 0$ with $0 \leq h+i \alpha \leq d$. Moreover, $a_{(i-1) \alpha+\beta}^{*} \leq a_{i \alpha+\beta}^{*}$, and $a_{(i-1) \alpha+\beta+1}^{*} \geq a_{i \alpha+\beta+1}^{*}$.

Lemma 9 If $\alpha=2 \beta$ with $\alpha<d$, then $a_{i}^{*}=0$ for all $i$.

Proof: By Lemma 7, $a_{i}^{*}=0$ for all $i=0,1, \ldots, \alpha$. Hence we have the assertion by Lemma 8 (1).

Lemma 10 Suppose $\alpha=2 \beta+1$. If $a_{h}^{*}=0$ for all $h=0,1, \ldots, \alpha$. Then $a_{j}^{*} \neq 0$ only when $j=d$ and $d \equiv \beta \quad(\bmod \alpha)$.

Proof: Choose an integer $i$ so that $i \alpha+1 \leq j \leq(i+1) \alpha$. We prove by induction on $i$. There is nothing to prove when $i=0$.

By induction hypothesis and Lemma 8, we may assume that $j=i \alpha+\beta$ or $j=i \alpha+\beta+1$. Since $a_{(i-1) \alpha+\beta+1}^{*} \geq a_{i \alpha+\beta+1}^{*}, a_{i \alpha+\beta+1}^{*}=0$ by induction hypothesis.

Suppose $i \alpha+\beta<d$. Then $q_{(i-1) \alpha+\beta+2, i \alpha+\beta+1}^{\alpha}=0$ and $a_{(i-1) \alpha+\beta+1}^{*}=a_{i \alpha+\beta+1}^{*}$ implies $q_{(i-1) \alpha+\beta+1, i \alpha+\beta}^{\alpha}=0$ by Lemma 2 (4)(ii). Since $q_{(i-1) \alpha+\beta, i \alpha+\beta-1}^{\alpha}=0$, we have $0=a_{(i-1) \alpha+\beta}^{*}=a_{i \alpha+\beta}^{*}$ by Lemma $2(4)(i i i)$ as desired.

Lemma 11 Suppose $\alpha=2 \beta+1 \geq 5$ with $\alpha<d$. Then $a_{i}^{*}=0$ for all $i<d$. Moreover, $a_{d}^{*} \neq 0$ only if $d \equiv \beta \quad(\bmod \alpha)$.

Proof: By Lemma 10 and Lemma 7, it suffices to show that $a_{\beta+1}^{*}=0$.

Assume that $\alpha \geq 7$. Then we have

$$
a_{\beta+2}^{*}=\cdots=a_{\alpha}^{*}=a_{\alpha+1}^{*}=a_{\alpha+2}^{*}
$$


by Lemma $8(2)$ as $a_{1}^{*}=a_{2}^{*}=0$. Note that $d \geq \alpha+2$ by Lemma 5 (2). Since $d \geq \alpha+2$, we have by Lemma $2(3)$ that $a_{\beta+1}^{*}=0$ as $(\alpha+2)-(\beta+2)=\beta+1$.

Suppose $\alpha=5$. Then we have

$$
a_{1}^{*}=a_{2}^{*}=a_{4}^{*}=a_{5}^{*}=a_{6}^{*}=0 .
$$

Now $q_{2,4}^{5}=0$ by Lemma 6 . Since $q_{2,5}^{4}=0$ and $a_{2}^{*}=a_{6}^{*}$, by Lemma $2(4)(i)$, we have $q_{3,6}^{4}=0$. Hence $q_{4,3}^{6}=q_{1,6}^{6}=0$, and by Corollary $1(1), a_{3}^{*}=0$ as $d \geq 7$ by Lemma 5 (2).

Lemma 12 Suppose $\alpha=3<d$. Then one of the following holds.

(1) $a_{i}^{*}=0$ for all $i<d$, and $a_{d}^{*} \neq 0$ only if $d \equiv \beta \quad(\bmod 3)$.

(2) $d=6, a_{1}^{*}=a_{3}^{*}=a_{6}^{*}=0$, and $a_{2}^{*}=a_{4}^{*}+a_{5}^{*} \neq 0$.

(3) $d=4, a_{1}^{*}=a_{3}^{*}=0$, and $a_{2}^{*} \neq 0$.

Proof: It is easy to see that $a_{1}^{*}=a_{3 i}^{*}=0$ for every $i$. Suppose $d \geq 7$. Since $q_{3,4}^{6}=0$, $a_{4}^{*}=0$ by Corollary 1 (1). Since $q_{1,3}^{3}=0$ and $a_{1}^{*}=a_{4}^{*}=0, q_{2,4}^{3}=0$ and $a_{2}^{*}=a_{5}^{*}$ by Lemma 2 (4) (i), (iii). Moreover, $q_{3,2}^{4}=0$ with $a_{4}^{*}=0$ implies $a_{2}^{*}=0$ by Corollary 1 (1). Therefore we have $a_{1}^{*}=a_{2}^{*}=a_{3}^{*}=0$. Hence we have (1) in this case.

If $d \leq 6$, then $d=4$ or 6 by Lemma 5 (2). If $d=4$ or 6 , then we have $a_{1}^{*}=a_{3 i}^{*}=0$ for every $i$. Moreover, since $q_{3,3}^{5}=0, a_{4}^{*}+a_{5}^{*}=a_{2}^{*}$. Clearly if $a_{2}^{*}=0$, we have case (1) by Lemma 10. Hence we have one of the three cases above.

Lemma 13 Suppose $d \geq \alpha+2$ with $\alpha>2$ and $a_{i}^{*}=0$ for all $i=1,2, \ldots, d-1$. Then $k_{1}^{*}=2$.

Proof: Suppose $k_{1}^{*}>2$. Observe by Lemma 6 that $q_{\alpha-h+2, h}^{\alpha}=0$, unless $\alpha=2 \beta$ and $h=\beta+1$ for $2 \leq h \leq \alpha$. Moreover $q_{2, \alpha}^{\alpha}=q_{2, \alpha-1}^{\alpha}=0$ by our assumption. Hence by Corollary $1(2), q_{2, h}^{h}=0$ when $q_{\alpha-h+2, h}^{\alpha}=0$.

Suppose $\alpha=2 \beta+1$. Then $q_{2, h}^{h}=0$ for $2 \leq h \leq \alpha$. Hence

$$
c_{h}^{*} b_{h-1}^{*}+b_{h}^{*} c_{h+1}^{*}-k_{1}^{*}=0 .
$$

Now by induction we show that $c_{h}^{*} \leq 1$ when $h$ is odd. The assertion is trivial if $h=1$. Suppose $c_{h-1}^{*} \leq 1$. Since $q_{2, h}^{h}=0$, we have

$$
\begin{aligned}
0 & =c_{h}^{*} b_{h-1}^{*}+b_{h}^{*} c_{h+1}^{*}-k_{1}^{*} \\
& =c_{h}^{*}\left(k_{1}^{*}-c_{h-1}^{*}\right)+b_{h}^{*} c_{h+1}^{*}-k_{1}^{*} \\
& \geq c_{h}^{*}\left(k_{1}^{*}-1\right)+b_{h}^{*}+b_{h}^{*}\left(c_{h+1}^{*}-1\right)-k_{1}^{*} \\
& \geq c_{h}^{*}+b_{h}^{*}-k_{1}^{*}+b_{h}^{*}\left(c_{h+1}^{*}-1\right) \\
& \geq b_{h}^{*}\left(c_{h+1}^{*}-1\right)
\end{aligned}
$$


Thus $c_{h+1}^{*}-1 \leq 0$. Since $\alpha$ is odd, $c_{\alpha-2}^{*} \leq 1$. Now by Proposition $1, b_{2}^{*}=c_{\alpha-2}^{*} \leq 1$. Note that this holds for $\alpha=5$ as well because $a_{2}^{*}=0$ in our case. Since $q_{2,2}^{2}=0$, we have

$$
0=c_{2}^{*} b_{1}^{*}+b_{2}^{*} c_{3}^{*}-k_{1}^{*}>c_{2}^{*} b_{1}^{*}-k_{1}^{*} \geq\left(k_{1}^{*}-1\right)^{2}-k_{1}^{*} .
$$

This is impossible. Hence we have the assertion when $\alpha$ is odd.

Suppose $\alpha=2 \beta$. Then the argument above shows that $c_{h}^{*} \leq 1$ when $h$ is odd and $h \leq \beta+1$. Note that $q_{2, \beta}^{\beta}=0$. Suppose $h$ is odd and $h \leq \beta$. Then

$$
0=c_{h}^{*} b_{h-1}^{*}+b_{h}^{*} c_{h+1}^{*}-k_{1}^{*}>\left(k_{1}^{*}-1\right) c_{h+1}^{*}-k_{1}^{*} .
$$

Since $k_{1}^{*} \geq 3$ as $k_{1}^{*}$ is an integer, $c_{h+1}^{*}<3 / 2$. Therefore, we have the following.

$c_{h}^{*} \leq 1$ if $h$ is odd and $h \leq \beta+1$.

$c_{h}^{*}<3 / 2$ if $h$ is even and $h \leq \beta+1$.

Suppose $\beta$ is odd. Then $b_{\beta-1}^{*}=c_{\beta+1}^{*}<3 / 2$ by Proposition 1 . Since $c_{\beta-1}^{*}<3 / 2$, $3 / 2>b_{\beta-1}^{*}=k_{1}^{*}-c_{\beta-1}^{*}>k_{1}^{*}-3 / 2$. Thus $k_{1}^{*}<3$. This contradicts our assumption.

Suppose $\beta$ is even. Then $c_{\beta-1}^{*} \leq 1$ and $b_{\beta-1}^{*}=c_{\beta+1}^{*} \leq 1$. Thus we obtain that $k_{1}^{*} \leq 2$.

This proves the assertion.

Proof of Theorem 3: Suppose $\alpha=2$. Then by Lemma 9, we have $(i)$. Suppose $\alpha=d$. Then by Proposition 1, we have (ii). Suppose $2<\alpha<d$. If $\alpha$ is even, then $a_{i}^{*}=0$ for every $i$ by Lemma 9 . If $\alpha$ is odd, then by Lemma 11, Lemma 12 and Proposition 1, we have $a_{i}^{*}=0$ for all $i=1,2, \ldots, d-1$ unless $\alpha=3$ and (iii) or $(i v)$ holds. Now by Lemma 13 , we cannot have other cases.

This completes the proof of Theorem 3.

\section{Acknowledgments}

The author is very grateful to Dr. Garth A. Dickie for sending him preprints. The author learned a lot from these papers. He is also grateful to Dr. Masato Tomiyama for his careful reading of the manuscript. Dr. Tomiyama pointed out a serious gap in the first manuscript. The author also would like to thank the anonymous referee who greatly improved the clarity of this paper by providing many pages of helpful comments.

\section{References}

1. E. Bannai and T. Ito, Algebraic Combinatorics I, Benjamin-Cummings, California, 1984.

2. A. E. Brouwer, A. M. Cohen and A. Neumaier, Distance-Regular Graphs, Springer Verlag, Berlin, Heidelberg, 1989.

3. P. J. Cameron, J. M. Goethals and J. J. Seidel, "The Krein condition, spherical designs, Norton algebras and permutation groups," Indag. Math. 40 (1978), 196-206.

4. G. A. Dickie, " $Q$-polynomial structures for association schemes and distance-regular graphs," Ph.D. Thesis, University of Wisconsin, 1995.

5. G. A. Dickie, "A note on $Q$-polynomial association schemes," preprint. 